Ann. Abeille, I968, 11 (3), I3I-I49.

\title{
ÉTUDE ÉCONOMIQUE DE QUELQUES EXPLOITATIONS APICOLES DU BASSIN PARISIEN
}

\author{
D. DURIS \\ École nationale supérieure agronomique, 78 - Grignon \\ Institut national de la Recherche agronomique
}

SOMMAIRE

Vingt at une exploitations apicoles à caractère professionnel, toutes situées au nord de la Loire, ont fait l'objet d'une étude économique reposant sur l'examen de la comptabilité des entreprises et sur les renseignements fournis par les apiculteurs ayant accepté l'enquête. Les résultats obtenus montrent la grande variabilité des produits, des marges et des revenus des apiculteurs, le poids important des charges fixes et spécialement des charges de travail dans l'ensemble des charges, l'effet d'échelle peu marqué sur l'économie des charges ainsi que l'incidence considérable sur les résultats économiques des formes de production et des modes de commercialisation.

Tout jugement sur la profession apicole ne peut donc être que nuancé. Fin dépit des difficultés actuelles résultant de la faiblesse des prix de vente du miel, il existe encore des situations présentant des rentes appréciables de production ou de commercialisation.

\section{IN'TRODUCTION}

Depuis plusieurs années la situation économique de l'apiculture se dégrade progressivement. Les difficultés semblent d'ailleurs être plus graves chez les professionnels que chez les amateurs.

Les causes fondamentales de ces difficultés économiques sont à rechercher dans la stagnation des prix de vente du miel à la production, principalement sous l'influence d'une indéniable sous-consommation et d'une concurrence très forte exercée par les miels d'importation en provenance de pays plus favorisés ou disposant d'une main-d'œuvre peu coûteuse.

Si le malaise de l'apiculture française est réel, il n'en est pas moins vrai que nous ne disposons pas d'éléments chiffrés sérieux permettant de connaître avec précision a situation financière des exploitations. Il nous a donc semblé que seule une analyse 
détaillée des comptes d'un certain nombre d'exploitations nous permettrait de détecter les points faibles et de proposer des solutions aux problèmes qui se posent, si toutefois de telles solutions existent.

Grâce à l'appui du Syndicat des Producteurs de Miel de France nous avons pu enquêter dans 2 I exploitations apicoles importantes, toutes situées au nord de la Loire. C'est le résultat de cette enquête que nous livrons ci-dessous avec l'autorisation du S. P. M. F.

\section{MÉTHODES}

\section{A. - Choix des apiculteurs}

Le nombre de personnes visitées est de $2 \mathrm{r}$. Ce nombre est un peu faible mais il n'a pas été possible de l'augmenter. Le choix a porté sur des personnes dont l'apiculture est l'activité unique ou principale. L'échantillonnage est assez représentatif du point de vue statistique puisqu'il y a 35 p. roo des apiculteurs professionnels connus dans les départements touchés par l'enquête qui ont été contactés.

\section{B. - Mode d'enquête}

L.es enquêtes ont été faites sous la forme d'un entretien personnel de plusieurs heures avec: chaque apiculteur. Les questions ont porté sur les structures de l'exploitation, sur les méthodes de travail, sur les produits et sur les résultats financiers obtenus. Dans de nombreux cas on a pu consulter les documents comptables.

\section{C. - Zones d'enquêtes}

On peut diviser la région géographique soumise à l'enquête en 6 zones très bien individualisées :

$\begin{array}{ll}\text { Brie et Champagne, } & \text { Gâtinais, } \\ \text { Jura, } & \text { Champagne berrichonne, } \\ \text { Beauce et plaine de Versailles, } & \text { Val de Loire (Anjou et Orléanais). }\end{array}$

\section{D. - Difficultés rencontrées}

Ces difficultés sont essentiellement d'ordre analytique. Pour les diminuer on a essayé d'étudier uniquement la campagne 1967-68 (récolte I967). Cependant, il n'a pas toujours été facile de connaître les charges afférentes à cette campagne car beaucoup d'apiculteurs ne tiennent pas de comptabilité.

Par comparaison avec des apiculteurs tenant une comptabilité très sérieuse, il a été possible de vérifier la valeur des renseignements fournis par les autres. Fin règle générale, on peut estimer qu'ils sont valables avec une marge d'erreur de io p. ioo environ.

Par contre, pour le tonnage des différentes productions, il est plus difficile de connaître l'erreur commise. Tout d'abord, l'exercice étudié correspond à une bonne année et les apiculteurs visités ont souvent eu de bons résultats. Il semble cependant d'après les renseignements obtenus que les tonnages soient valables à environ to p. 100 près.

Tinfin, les prix indicjués peuvent être retenus car les "fourchettes " de prix sont toutes les mêmes. Il n'y a guère que le prix de vente moyen qui puisse être discuté car il est impossible de connaître les quantités vendues à chacun des prix donnés. La marge globale d'erreur doit être nalgré tout asie\% faible. 


\section{RÉSULTATS}

\section{Données générales}

a) Situation des exploitations.

Le nombre des apiculteurs visités dans chacune des régions a été :

Brie : 5
Champagne : I
Gâtinais : 4
Orléanais : 2

Anjou : 2

Beauce et plaine de Versailles : 4

Champagne berrichonne: I

Jura et Dombes : 2

b) Flore mellifère.

Tous les apiculteurs visités récoltent du miel de Colza. Certaines régions fournissent des miels de Sapin, de Bruyère et de Sainfoin. On trouve également des miels "toutes fleurs".

c) Le rucher. Le matériel. Les battiments.

Sur 2 I apiculteurs, 8 ne pratiquent aucune transhumance. Les ruches sont donc sédentaires et les emplacements sont situés dans un rayon de I5 à $40 \mathrm{~km}$ autour du bâtiment d'exploitation.

Parmi les apiculteurs qui pratiquent la transhumance on distingue :

- les apiculteurs qui ne dépassent pas un rayon de roo $\mathrm{km}$ autour de leur exploitation. Dans ce cas les ruches suivent surtout la floraison du Colza. Après la première récolte elles sont ramenées dans une zone assez proche de l'exploitation.

- Les apiculteurs qui n'hésitent pas à parcourir de grandes distances afin de suivre les floraisons (étalement dî̀ à des différences de climat) et de rechercher une flore particulièrement intéressante : Lavande, Sapin, Romarin.

Le type de ruches (tabl. I) utilisées est très variable d'une exploitation à l'autre et même à l'intérieur d'une même exploitation. La normalisation A.F.N.O.R. est appliquée par 3 apiculteurs.

TABIEEAU I

Types de ruches utilisées

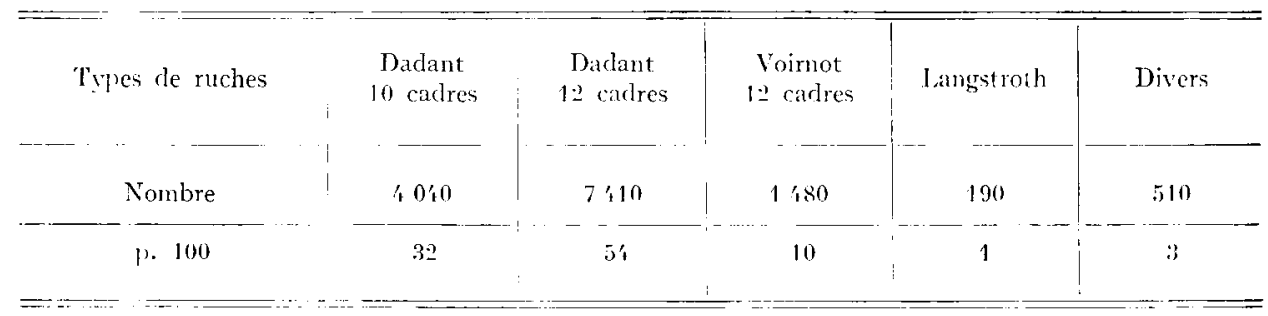

I a plupart de; ruches sont de type pastoral à toit plat et sans pied afin de faciliter la manutention. Celles qui ne servent pas à la transhumance sont à toit chalet. Il 
existe en outre environ 250 ruches paniers réservées à la production d'essaims. Les ruches sont en général assez vieilles (moyenne : I 8 ans).

Le matériel d'extraction et de stockage du miel est souvent âgé. Au cours des enquêtes, il n'a été rencontré que 6 apiculteurs ayant un matériel de moins de ro ans. Les autres ont un matériel dont l'âge varie de ro à 25 ans. Cependant, comme pour les ruches, ce matériel est solide et s'use peu. Pour les amortissements, il est raisonnable de les calculer sur une durée de 20 ans. Ils sont calculés en prenant ro p. Ioo de la valeur d'inventaire en début d'exercice.

Les bât 'ments sont généralement amortis (sauf chez 4 apiculteurs) car les exploitations exic tent depuis 2 ou 3 générations. Évidemment, il y eut, entre temps, un grand nombre de modifications.

Les véhicules rencontrés sont de deux types :

- véhicules lourds très vieux mais ayant peu roulé et servant à la transhumance sur de grandes distances (moyenne d'âge : I5 ans);

- véhicules légers servant pour les visites et le transport des hausses.

d) Les races d'abeilles.

Le tableau 2 donne l'indication des différentes races d'abeilles utilisées par les apiculteurs visités. On constate une assez grande diversité.

TABLEAU 2

Races des abeilles

\begin{tabular}{|c|c|}
\hline Races des abeilles et métis & Fréquence \\
\hline Italienne............ & 1 \\
\hline Abeille noire commune... & t \\
\hline Abeille noire $\times$ Italienne.... & 8 \\
\hline Abeille noire $\times$ Caucasienne... & 1 \\
\hline Ilalo-Caucasienne ................. & 1 \\
\hline Abeille noire $\times$ Italo-Caucasienne.. & 3 \\
\hline
\end{tabular}

e) Le mode d'exploitation.

Toutes les ruches sont en faire-valoir direct, sauf quelques-unes qui ont été achetées en viager à des apiculteurs âgés. Dans ce cas le montant de la rente viagère s'élève à environ $7,50 \mathrm{~F}$ par ruche, ce qui correspond au produit de 3 à $4 \mathrm{~kg}$ de miel par ruche estimé au prix de gros ( $\mathrm{I} / 2$ à $\mathrm{I} / 4$ de la production par ruche suivant les années).

Deux apiculteurs louent leurs ruches à des agriculteurs pour la pollinisation à raison de 35 à $40 \mathrm{~F}$ la ruche.

\section{Conduite du rucher}

a) Les colonies.

Il y a chaque année des pertes de colonies par orphelinage, mort hivernale, essaimage, maladies. Tous les apiculteurs consacrent quelques ruches au remplace- 
ment des essaims perdus. Les pertes peuvent varier d'une exploitation à l'autre. Elles dépendent de la méthode de conduite du rucher et des soins apportés aux abeilles. La moyenne est de 6 p. Ioo (entre 3 et ro p. Ioo).

Quelques apiculteurs pratiquent l'élevage de reines ( 8 sur $2 \mathrm{I}$ ), soit pour la vente, soit pour le renouvellement.

Neuf apiculteurs marquent leurs reines et 5 seulement les changent tous les deux ans. Les autres ne les changent que si la ruche périclite.

\section{b) Les productions.}

Les productions sont très diversifiées en apiculture. Au cours de l'enquête on a rencontré 9 apiculteurs faisant uniquement du miel, 2 apiculteurs faisant du pollen et du miel et ro apiculteurs faisant miel et gelée royale (dont 8 avec pollen).

Le miel. - Production totale : 240 tonnes, chez les apiculteurs visités. Pour la campagne 1967 , qui fut bonne, les rendements ont varié de $\mathrm{I} 2$ à $28 \mathrm{~kg}$ suivant les exploitations. Le rendement moyen pour I I 900 ruches a été de 20, I $\mathrm{kg}$; le rendement par région a été le suivant :

$\begin{array}{ll}\text { Brie : I9 kg } & \text { Champagne : } 28 \mathrm{~kg} \\ \text { Jura : I5 kg } & \text { Val de Loire : } 13 \mathrm{~kg} \\ \text { Gâtinais-Beauce : } 2 \mathrm{r} \mathrm{kg} & \end{array}$

Six apiculteurs ont obtenu une moyenne de production supérieure à $20 \mathrm{~kg}$.

Les dates de récolte du miel varient beaucoup. Elles dépendent de la fin des floraisons. Dans tous les cas, la première récolte se situe vers le 20 mai, c'est-à-dire après la floraison du colza. En général, il y a une seconde récolte à la fin de juillet ou au début d'août, après la floraison des prairies. La récolte du miel de sapin se situe à partir du début d'août et peut se prolonger jusqu'en septembre, voire octobre.

Le pollen. - Production totale : $5935 \mathrm{~kg}$ chez les apiculteurs visités. Le rendement moyen est de $2,6 \mathrm{~kg}$ par ruche. Le rendement par exploitation varie de 0,5 à $4 \mathrm{~kg}$ suivant la région et suivant la durée de la pose des trappes à pollen. Celle-ci varie de 2 mois et demi à trois mois $\left(\mathrm{I} / 5-3 \mathrm{I} / 7, \mathrm{I} 5 / 4-\mathrm{I} 5 / 7, \mathrm{I} / 5^{-\mathrm{I}} 5 / 7\right)$. La production du pollen entraîne des frais assez élevés par suite des déplacements nécessaires.

La gelée royale. - Production totale : $270 \mathrm{~kg}$ chez les apiculteurs visités. Le rendement moyen est de $267 \mathrm{~g}$ par ruche utilisée pour cette production. Il varie de go à $35^{\circ} \mathrm{g}$. La période de production est plus ou moins longue ( $\mathrm{a}$ à 5 mois) avec une moyenne de 3 mois. En fait, la production est importante pendant le premier mois puis elle décroît rapidement. Les ruches produisant à la fois du miel et de la gelée royale ont des rendements faibles : 8 à Io $\mathrm{kg}$ de miel, 90 à Ioo $\mathrm{g}$ de gelée royale.

La production de la gelée royale demande beaucoup de travail et entraîne des dépenses élevées. La récolte doit se faire obligatoirement tous les trois jours, ce qui entraîne de fréquents déplacements.

\section{Résultats globaux}

a) Importance des exploitations (tabl. 3).

Les quatre entreprises de plus de $\mathrm{I}$ ooo ruches ont respectivement : 2200,2500 , I 800 et I ooo ruches. 
TABIEAU 3

Importance des exploitations visitées

\begin{tabular}{c|c|cc|c|c|c}
\hline Nombre de ruches & Moins de 300 & 300 à 500 & 500 à 750 & 750 à 1000 & Plus de l 000 \\
\hline Fréquence & 3 & 8 & 1 & t & t \\
\hline
\end{tabular}

b) Les produits bruts (tabl. 4).

Sauf pour les hydromeliers les produits bruts résultent des ventes des produits récoltés pendant la campagne considérée. L'exercice commence au I er mars 1967 et se termine au 29 février I968. A cette date les stocks étaient nuls ou minimes pour la plupart des exploitations. La date du I ${ }^{\mathrm{er}}$ mars correspond à une reprise d'activité.

TAIBI,EAU 4

Produits bruts totaux et par ruche

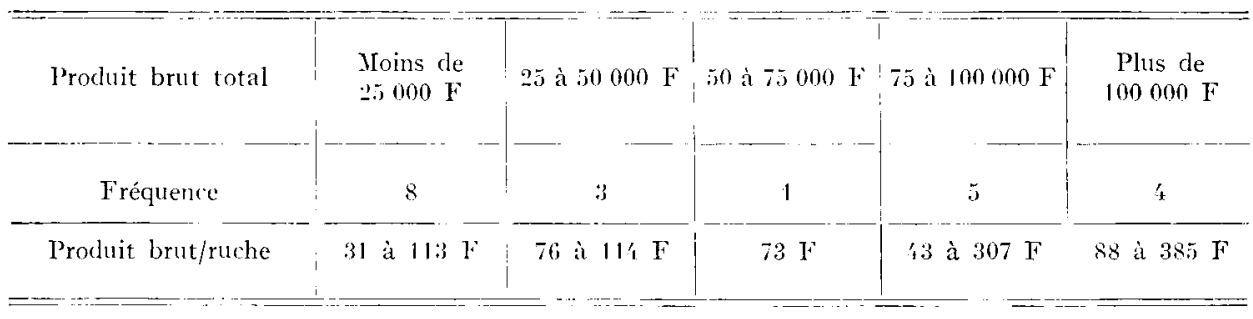

Le cas des hydromeliers est particulier. Les hydromels vendus pendant cet exercice sont ceux qui ont été fabriqués avec le miel des exercices antérieurs (récoltes de 1965 et I 966 ). En effet, il faut du temps pour obtenir une bonne fermentation et l'hydromel doit vieillir avant d'être commercialisé.

L'échelle des produits bruts est très étalée puisqu'elle va de I2 $000 \mathrm{~F}$ à $286000 \mathrm{~F}$. En premier lieu, on peut penser que ceci est dî uniquement au nombre de ruches des exploitations. Or, ramené à la ruche, le produit brut varie de 3 I à $385 \mathrm{~F}$ et ce, indépendamment des produits bruts totaux. Dans chaque classe, on constate l'existence de "fourchettes " importantes. Les causes sont sans doute multiples mais les deux principales paraissent être :

- la conduite de l'exploitation : technicité, moyens mis en œuvre par l'apiculteur, spéculations pratiquées,

- la commercialisation des produits et la transformation éventuelle du miel.

Ces deux causes seront étudiées plus loin lors de la discussion des résultats.

En résumé, on peut dire que le nombre de ruches a une importance certaine mais il serait illusoire de penser qu'il constitue le facteur principal d'une bonne rentabilité. Pour un mode de commercialisation donné il y a vraisemblablement une di- 


\section{TABIEAU 5}

Charges fixes et charges variables (par ruche)

Exploitation à une seule spéculation

\begin{tabular}{|c|c|c|c|c|c|c|c|c|c|}
\hline & & & & & & & & & \\
\hline Nombre de ulches....... & 250 & 270 & 300 & 300 & 350 & 390 & 600 & 600 & 1000 \\
\hline Charges fixes ...... & 40,55 & 18,30 & $\because 0,00$ & 205,95 & $18, \geq 0$ & 12,35 & 13,55 & 14,85 & 51,05 \\
\hline Charges variables $\ldots \ldots \ldots \ldots$ & 19,30 & 9,95 & 10,20 & 22,60 & 22,60 & 8,10 & 6,75 & 10,15 & 14,15 \\
\hline Main-d'œuvre $\ldots \ldots \ldots \ldots \ldots$ & $\mathrm{T}$ & $\mathrm{F}$ & $\mathrm{F}$ & $\mathrm{F}-\mathrm{T}$ & $\mathrm{F}-\mathrm{l}$ & Ii & $\mathrm{F}-\mathrm{P}$ & P-'T & F-P-T \\
\hline Commercialisation ........ & $1-2$ & 3 & $1 \because 2$ & $1 \cdot 1$ & 1 & 1- أ & i & 4 & $1-2 * a$ \\
\hline
\end{tabular}

\section{Exploitation à 2 spéculations}

\begin{tabular}{|c|c|c|c|c|}
\hline \multirow[b]{2}{*}{ Nombre de ruches ....... } & \multicolumn{2}{|c|}{ Miel-Pollen } & \multicolumn{2}{|c|}{ Miel-Crelée royale } \\
\hline & 5,0 & 430 & 150 & $\because 200$ \\
\hline Charges fixes........... & & & 40,80 & 46,69 \\
\hline Charges variables ....... & & & 26,50 & 3,95 \\
\hline Main-d'œuvre $\ldots \ldots \ldots \ldots$ & & & $I^{i}$ & $\mathbf{P}$ \\
\hline Commercialisation......... & $1-3-4$ & $1-2$ & $1-.1$ & 4 \\
\hline
\end{tabular}

Exploitation à 3 spéculations

\begin{tabular}{|c|c|c|c|c|c|c|c|c|}
\hline Nombre de ruches & 300 & 300 & 600 & 600 & 890 & 900 & 1800 & 2500 \\
\hline Charges fixes ........ & 12,31 & 21 & $1:, 90$ & 71,20 & 18,70 & 17,80 & 30,10 & 48,20 \\
\hline Charges variables... & 7,70 & 50,35 & 14,90 & 27,20 & $1: 3,50$ & 10,30 & $1 ' t, 60$ & 17.10 \\
\hline Main-d'œuvre . & $\mathbf{F}$ & F-T & $\mathrm{F}-\mathrm{T}$ & $\mathrm{F}-\mathrm{P}-\mathrm{T}$ & $\mathrm{F}-\mathrm{T}$ & $\mathrm{F}-\mathrm{T}$ & F-P-T & F-P.P \\
\hline Commercialisation & $1-2$ & $1-2-* a$ & $1-* a_{-}^{*} b$ & $1-2-* b$ & 1 & $1-3$ & 1-'́t & $1-2-3-* b$ \\
\hline
\end{tabular}

F : MO familiale

$\mathrm{P}: \mathrm{MO}$ permanente

$\mathrm{T}$ : MO temporaire
Commercialisation :

$*_{a}$ : Hydromel

*b : Miel à la gelće royale
1 : détail

$2: 1 / 2$ gros

$3:$ gros

4 : coopérative. 
mension optimum de l'exploitation. Les différentes spéculations ont également leur importance.

c) Les charges fixes (tabl 5).

On constate d'abord que les charges fixes réelles sont très différentes d'une exploitation à l'autre. Ceci est dû à deux facteurs qui sont :

- la présence ou non d'ouvriers permanents,

- 1'âge de l'exploitation (matériel amorti ou non).

Les charges fixes réelles varient de $\mathrm{I} 20300 \mathrm{~F}$ à $3700 \mathrm{~F}$. Dans ce dernier cas, tout le matériel est amorti mais pour établir des comparaisons valables il vaut mieux se servir des charges par ruche. Dans ce cas on trouve des différences assez importantes (de I $2,30 \mathrm{~F}$ à $74,20 \mathrm{~F}$ ). Comme on le verra plus loin, ce sont les salaires permanents qui sont la cause de cette différence.

d) Les charges variables (tabl. 5).

Ces charges sont généralement faibles. Il y a trois postes vraiment importants que l'on rencontre dans toutes les exploitations :

l'achat de sucre pour le nourrissement des abeilles,

les frais de carburant, qu'il y ait transhumance ou non, les frais de conditionnement.

Ces postes représentent respectivement 32,4 p. Ioo, 26,8 p. roo et 22,3 p. Ioo des charges variables.

Le poste salaire pour le personnel temporaire est peu important sauf dans deux exploitations. Ceci est dû̀ à l'emploi de la main-d'œuvre familiale au moment de la récolte.

Dans les deux exploitations qui emploient beaucoup de salariés temporaires, ces derniers travaillent de 3 à 6 mois (de mai à octobre) et pendant la morte-saison le chef d'exploitation et un ouvrier permanent suffisent à assurer le travail.

A ce niveau, on se rend compte du caractère saisonnier de l'apiculture. Il serait beaucoup plus intéressant de n'employer que de la main-d'œuvre temporaire; les charges totales diminueraient considérablement; mais il se pose un problème de main-d'œuvre, ce qui montre bien que l'apiculture est une activité agricole.

\section{e) Les charges calculées.}

Sauf rares exceptions, le travail n'est pas réellement rémunéré. Les exploitants qui se versent un salaire se paient 6 à 7 F 1'heure. Par analogie, les salaires familiaux sont comptés sur la même base.

Tous les apiculteurs visités sont propriétaires de leurs bâtiments. Les loyers sont variables d'une région à une autre mais il semble raisonnable d'en estimer la valeur à I $500 \mathrm{~F}$ par an environ.

Les charges fixes calculées sont élevées pour les exploitations familiales sans ouvriers (permanents ou temporaires). Pour les charges variables calculées, elles sont toutes les mêmes, à savoir I,75 F par essaim (intérêt du cheptel vif) qui est le prix couramment pratiqué. 


\section{DISCUSSION}

\section{Analyse du produit brut}

Il n'est pas tenu compte des ventes de cire qui ne représentent qu'une faible partie du produit brut.

I. Exploitations ne produisant que du miel (tabl. 6).

Ces exploitations représentent un fort pourcentage (9 sur $2 \mathrm{I}$ ). La production totale atteint 70 tonnes avec 3730 ruches, soit un rendement moyen de $18,7 \mathrm{~kg}$.

\section{TABLEAU 6}

Produits bruts des exploitations à une seule spéculation (miel)

\begin{tabular}{|c|c|c|c|c|c|c|c|c|c|c|}
\hline Nombre de ruches ... & 250 & 270 & 300 & 300 & 350 & 390 & 600 & 600 & 1000 & Moy. \\
\hline Rendenrent $\mathrm{kg} / \mathrm{ruche}$ & 20 & 15 & $1:$ & 2.3 & 13 & $16 i$ & 13 & 14 & 28 & 18,7 \\
\hline Transhumance ...... & peu & non & non & peu & non & peu & non & non & oui & \\
\hline Produit brut/ruche... & 110,60 & 14,65 & 55,30 & 77,70 & 81,80 & 16,10 & 30,60 & 36,20 & $90, \geq 5$ & 63,70 \\
\hline Commercialisation . & $1-2$ & 3 & $1-\ddot{2}$ & $1-1$ & 1 & $1-1$ & t & 4 & $1-2-I I$ & \\
\hline
\end{tabular}

Commercialisation : 1 : détail

$2: 1 / 2$ gros

$3: \operatorname{gros}$

s: coopérative

II : vente d'hydromel.

Le produit brut total s'élève à $253000 \mathrm{~F}$, soit un prix moyen de $3,60 \mathrm{~F}$ par $\mathrm{kg}$ de miel. Toutefois, il faut retirer le miel transformé en hydromel (ce miel est vendu deux ans plus tard) ce qui donne un prix de vente moyen de $3,20 \mathrm{~F}$ par $\mathrm{kg}$ (production commercialisée directement : 63 tonnes pour une valeur globale de 202 ooo F).

On voit déjà que la commercialisation joue un rôle important dans la rentabilité de 1'apiculture.

Il est intéressant également de connaître les produits bruts par ruche et d'apprécier leur valeur d'après la dimension et le mode de commercialisation. Le produit brut est de $63,70 \mathrm{~F}$ par ruche en moyenne, mais il varic de $30,60 \mathrm{~F}$ à rio,6o F. Les exploitations qui ont le plus fort produit brut ne sont pas forcément les plus importantes. Déjà, à ce stade, on distingue 3 niveaux d'importance qui sont :

les exploitations de 300 ruches,

les exploitations de 600 ruches,

les exploitations de $\mathrm{I}$ ooo ruches et plus. 
A chacun de ces niveaux il est possible de classer les exploitations suivant le produit brut.

Le mode $\mathrm{d} s$ commercialisation est le facteur primordial. A chacun des niveaux définis plus haut on se rend compte que la vente au détail permet un produit brut élevé. On peut remarquer qu'entre les niveaux d'importance le produit brut par ruche varie. Ceci s'explique surtout par les tonnages relatifs vendus au détail et à la coopérative. Plus la quantité de miel produite est grande, plus il devient difficile de commercialiser au détail.

\section{Exploitations pratiquant plusieurs spéculations.}

Il s'agit essentiellement du pollen et de la gelée royale.

a) Miel et gelée royale (tab1. 7).

Il n'y a que deux apiculteurs pratiquant ces deux spéculations. La production totale de miel est de 32 tonnes et celle de gelée royale de I $5 \mathrm{~kg}$, soit des rendements moyens respectifs de I $5 \mathrm{~kg}$ de miel et de ir $5 \mathrm{~g}$ de gelée royale. Les produits bruts par

\section{TABLEAU 7}

Produits bruts des exploitations à deux spéculations

(miel-gelée royale) (miel-pollen)

\begin{tabular}{|c|c|c|c|c|}
\hline \multirow[b]{2}{*}{ Nombre de ruches..... . } & \multicolumn{2}{|c|}{ Miel-Celée royale } & \multicolumn{2}{|c|}{ Miel-Pollen } \\
\hline & 150 & 2200 & 540 & 130 \\
\hline Transhumance........... & non & oui & non & non \\
\hline Dates de récolte......... & $1-5-31-8$ & $20-4-15-7$ & $1-5-15-7$ & $15-15-15-7$ \\
\hline Froduit brut/ruche...... & 113,35 & 50,50 & 75,71 & 164 \\
\hline Commercialisation & $1-2$ & 4 & $1-3-4$ & $1-2$ \\
\hline
\end{tabular}

ruche sont, pour chacune de ces exploitations, de II3,35 F et de 50,50 F. Dans ce cas, les différences sont dues uniquement à la commercialisation. La première exploitation pratique uniquement la vente au détail et en demi-gros ; la seconde appartient à une coopérative.

Néanmoins, la gelée royale est une spéculation qui permet d'augmenter sensiblement le produit brut d'une exploitation. En effet, même à $0,90 \mathrm{~F}$ le gramme, la vente de I $\mathrm{kg}$ de gelée royale donne un même chiffre d'affaire que $360 \mathrm{~kg}$ de miel à $2,50 \mathrm{~F}$ avec un moindre nombre de ruches.

b) Miel et pollen.

Ces deux spéculations sont pratiquées par deux apiculteurs. Les productions sont de $700 \mathrm{~kg}$ de pollen et de I9 tonnes de miel. Le rendement moyen en pollen est de $2,750 \mathrm{~kg}$ par ruche. On retrouve toujours les problèmes de commercialisation. Le prix du pollen varie de 8 à $35 \mathrm{~F}$ par kg. Cette spéculation est intéressante. 
c) Miel, pollen et gelée royale (tabl. 8).

Ces trois spéculations sont pratiquées par 8 apiculteurs qui produisent :

- II9 tonnes de miel (50 p. Ioo de la production des apiculteurs visités),

$-5235 \mathrm{~kg}$ de pollen ( $88 \mathrm{p}$. Ioo de la production des apiculteurs visités),

- $255 \mathrm{~kg}$ de gelée royale (95 p. roo de la production des apiculteurs visités).

TABLEAU 8

Produits bruts des exploitations à 3 spéculations

\begin{tabular}{|c|c|c|c|c|c|c|c|c|}
\hline Nombre de ruches ... & 300 & 300 & 400 & 600 & 890 & 900 & 1800 & 2500 \\
\hline Transhumance ...... & peu & peu & oui & oui & oui & oui & oui & oui \\
\hline Produit brut........ & 34000 & 92000 & 154000 & 130800 & 80300 & 65250 & 164700 & 286400 \\
\hline Produit brut par ruche. & 114,20 & 307 & 385 & 218 & 91 & 73,0 & 88,80 & 114,60 \\
\hline Commercialisation & $1-3$ & $1-2-* a$ & $1-* a * b$ & $1-2-* b$ & 4 & $s^{\prime}$ & $1-1$ & $1-2-3-* b$ \\
\hline
\end{tabular}

On constate tout d'abord que les produits bruts sont élevés et que les produits par ruche sont plus forts que ceux rencontrés précédemment, même dans le cas de la vente à une coopérative. Le produit brut moyen pour les 8 exploitations est de I3I F par ruche.

Dans cette catégorie on trouve les exploitations les plus importantes, celles qui font de la transhumance sur de longues distances et où lej rendements sont les plus forts.

Ici, il est donc intéressant de connaître la répartition des revenus. Sauf les trois exploitations marquées d'un astérisque on constate que les produits bruts les plus forts sont obtenus avec une vente de gelée royale assez grande.

Les trois exploitations marquées d'un astérisque vendent soit de 1'hydromel (*a) soit un mélange de $\mathrm{s} 20 \mathrm{~g}$ de miel avec $2 \mathrm{~g}$ de gelée royale $(* b)$. Toutes les exploitations ont un réseau commercial très développé et celles qui commercialisent par une coopérative cherchent à développer les spéculations les plus intéressantes.

On remarque également que le nombre de ruches des entreprises est sensiblement un multiple de 300. Ceci laisse supposer que 300 ruches constituent une unité de production quelle que soit la spéculation.

Pour toutes les exploitations apicoles les techniques de production semblent jouer un rôle secondaire. On ne trouve pas de différence liée au type de ruches, à la race d'abeilles ou aux techniques employées. Ceci peut s'expliquer par l'insuffisance des informations recueillies au cours de l'enquête. Le problème qui se pose à l'apiculture semble être avant tout un problème de commercialisation.

Enfin, si l'unité de production paraît être de 300 ruches, seule l'analyse des charges peut permettre de savoir si une telle exploitation est viable ou non, indépendanment de la commercialisation. 


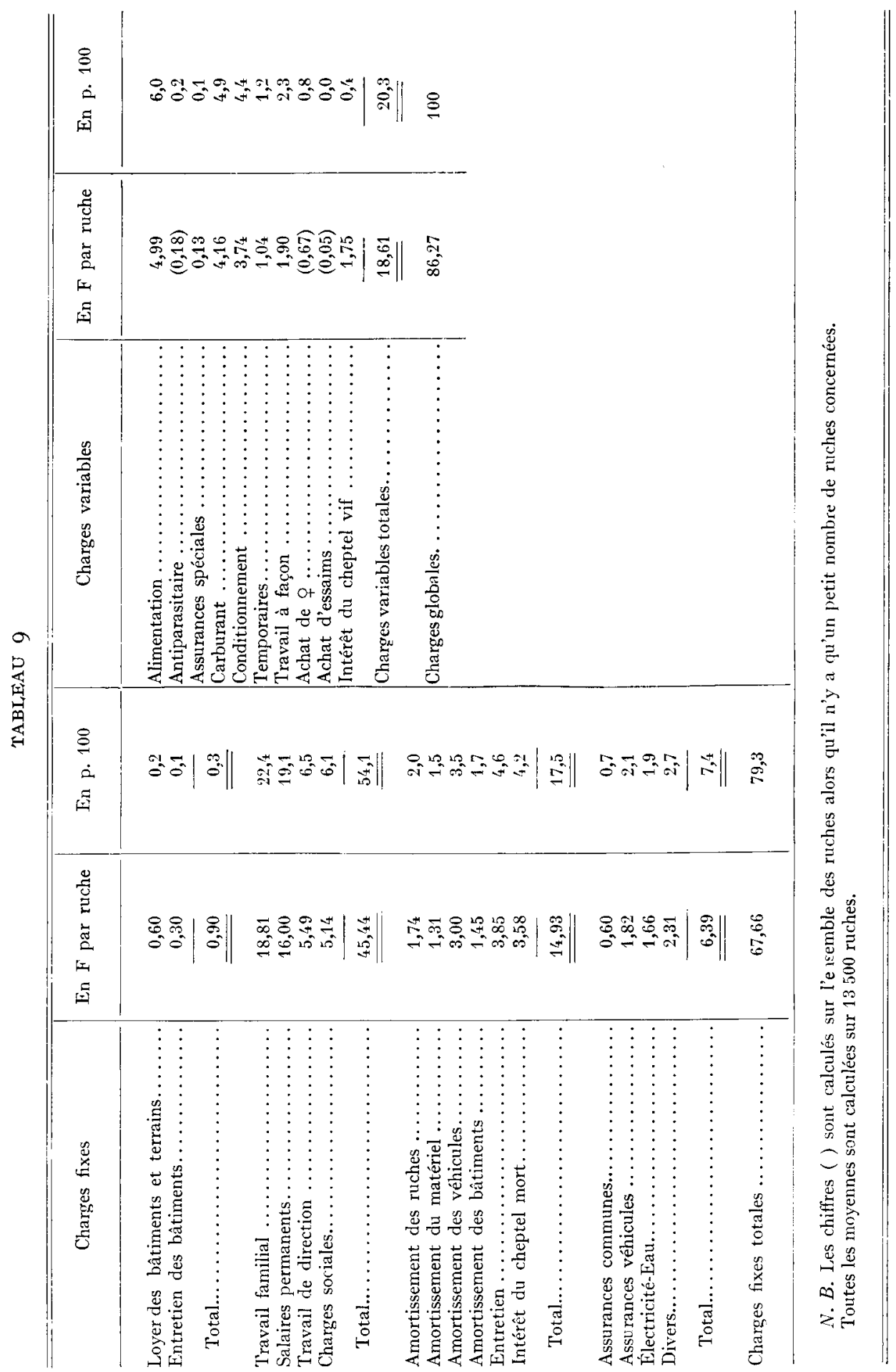




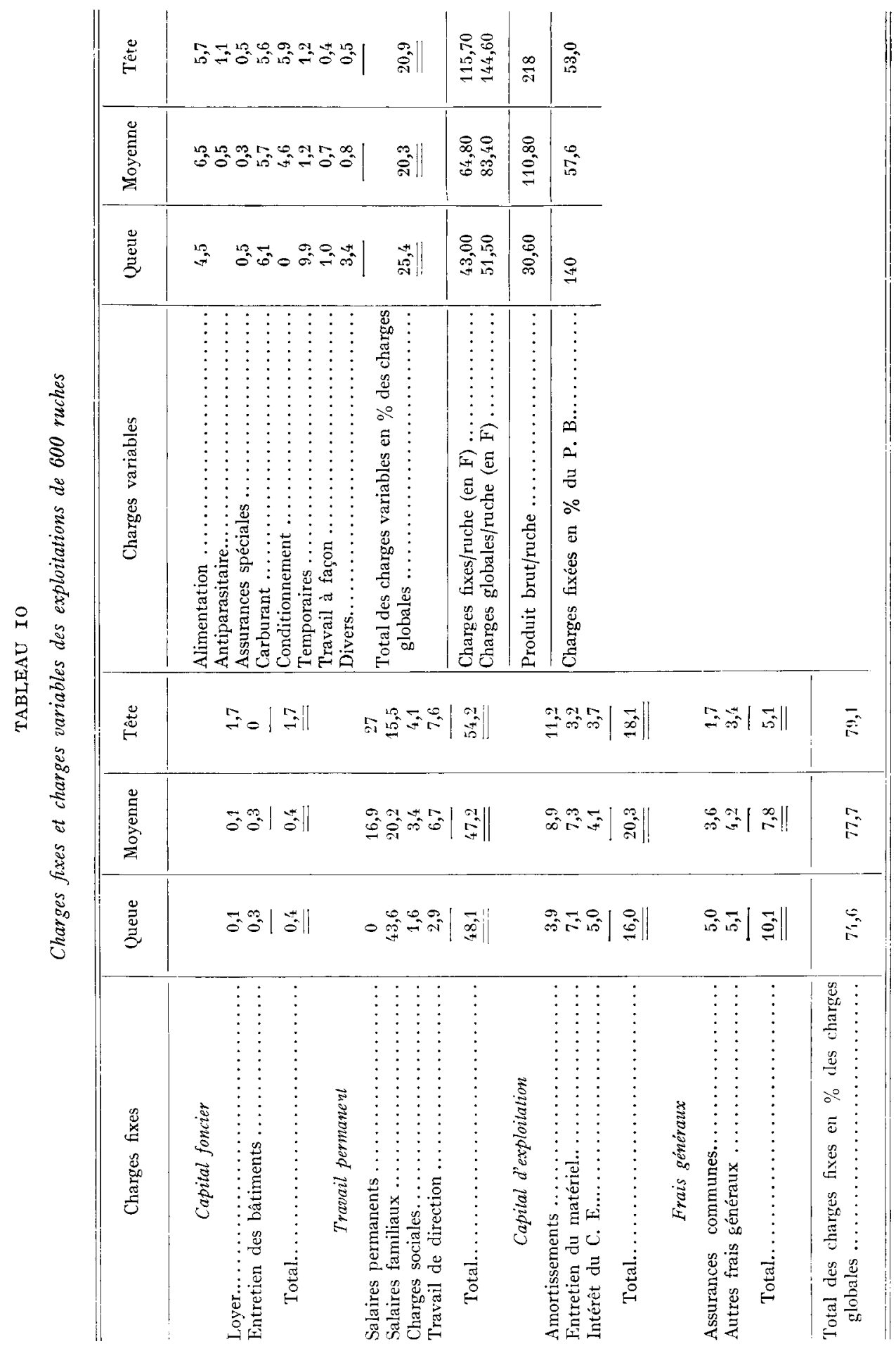




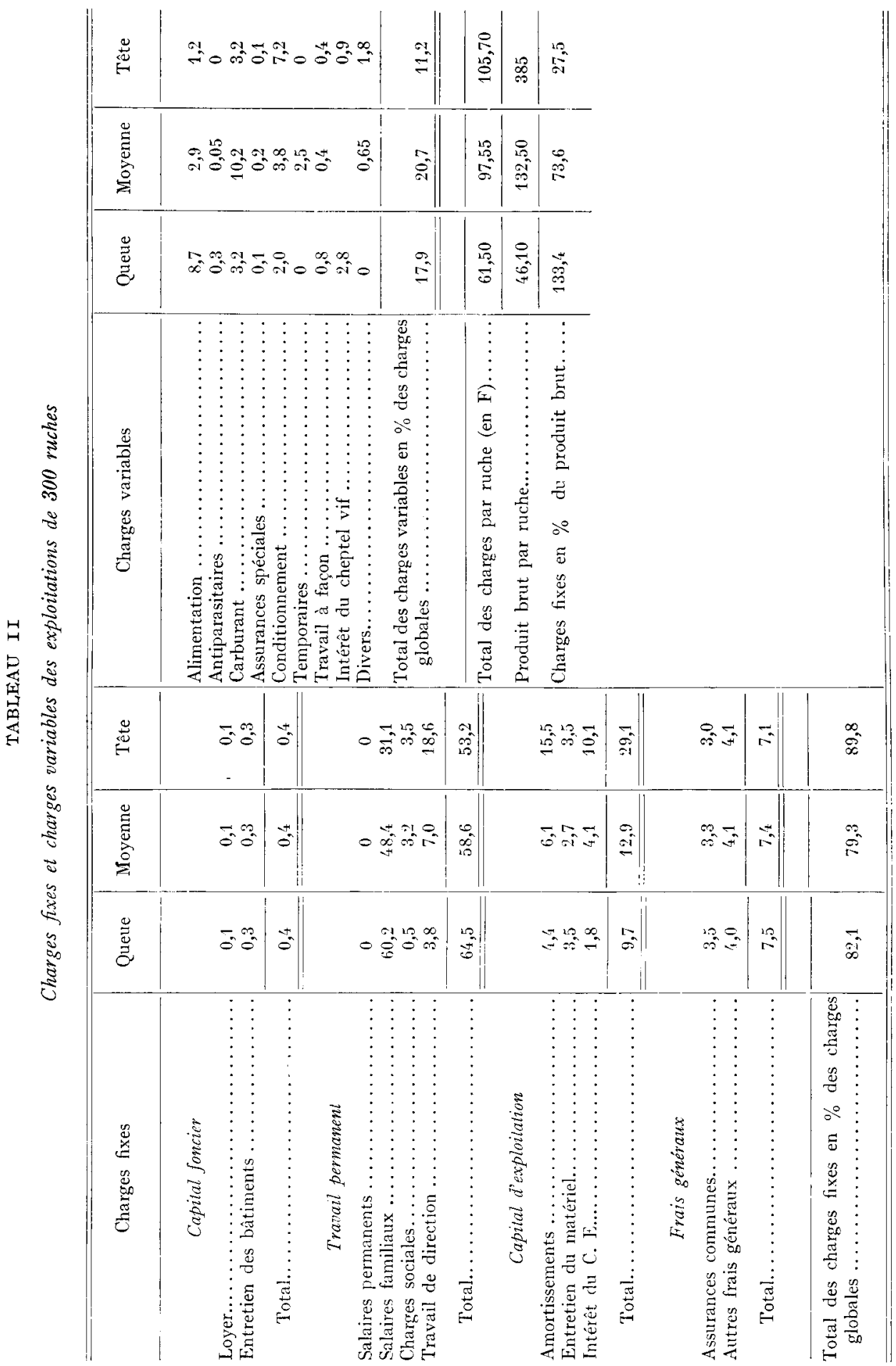




\section{Analyse des charges}

I. Charges fixes (tabl. 9, IO, II).

Pour l'ensemble des exploitations les charges fixes représentent 79,3 p. Ioo des charges globales. Parmi les charge; fixes les charges de travail sont les plus importantes (54, I p. Ioo des charges globales).

Parmi les charges calculées, le travail familial est le plus cô̂teux $(22,4$ p. IOO). A ce sujet il convient de faire la distinction entre apiculteurs ayant un ou plusieurs ouvriers et apiculteurs travaillant seuls. Chez ces derniers, le travail familial représente jusqu'à 5o p. Ioo des charges globales (moyenne : 38,9 p. IOo).

On constate donc que l'apiculture est une activité où les charges de main-d'œuvre sont élevées.

2. Charges variables (tabl. 9, ro, II).

Les charges variables ne représentent que 20,3 p. Ioo des charges globales. Les postes les plus importants sont l'alimentation des abeilles $(6,0 \mathrm{p}$. Ioo), le carburant (4,9 p. IOo) et le conditionnement (4,4 p. IOO).

Cependant, on peut noter qu'il est difficile de faire une analogie avec les charges variables en agriculture. En effet, en apiculture, les charges variables n'influent pas forcément sur la production. Par exemple, le nourrissement des abeilles sert uniquement à maintenir en bonnes conditions le cheptel vif mais, augmenter la quantité de sucre distribuée n'entraîne pas une meilleure production, tout au moins de façon systématique.

Les dépenses de carburant peuvent, dans une certaine mesure, conditionner le produit brut. En effet, la transhumance permet de rechercher une flore particulière qui donnera un miel unifloral qui sera vendu plus cher.

\section{Les résultats}

I. Marge brute (tab1. I2).

Compte tenu de l'importance des charges fixes le calcul de la marge brute n'est pas suffisant pour déterminer la rentabilité des exploitations apicoles.

2. Le revenu apicole (tabl. I2).

Le revenu moyen par ruche est de $49,5^{\circ} \mathrm{F}$. Ce revenu est assez faible. Il varie d'une exploitation à une autre et on constate que les grosses entreprises qui utilisent un ou plusieurs salariés permanents ont un revenu relativement faible (40 $\mathrm{F}$ par ruche).

3. Le bénéfice.

Le bénéfice moyen pour l'ensemble des exploitations est de I9,50 F par ruche. Or, on trouve que 12 exploitations sur $2 \mathrm{r}$ sont en perte. Cela s'explique, en partie, par l'estimation des salaires calculés de la main-d'œuvre familiale, en partie par la commercialisation et par le choix des productions.

4. Taux de rentabilité du capital d'exploitation.

Le taux moyen est de 5,3 p. Ioo. L'apiculture semble être une activité qui assure une rentabilité des capitaux tout juste suffisante. Cependant, il convient de voir dans 
quelle fourchette peut varier ce taux ; pour l'exploitation de tête, il est de I 8 p. Ioo et pour la plus mauvaise il est de 3,5 p. Ioo.

TABLEAU I2

Marge brute - Revenu apicole - Marge nette

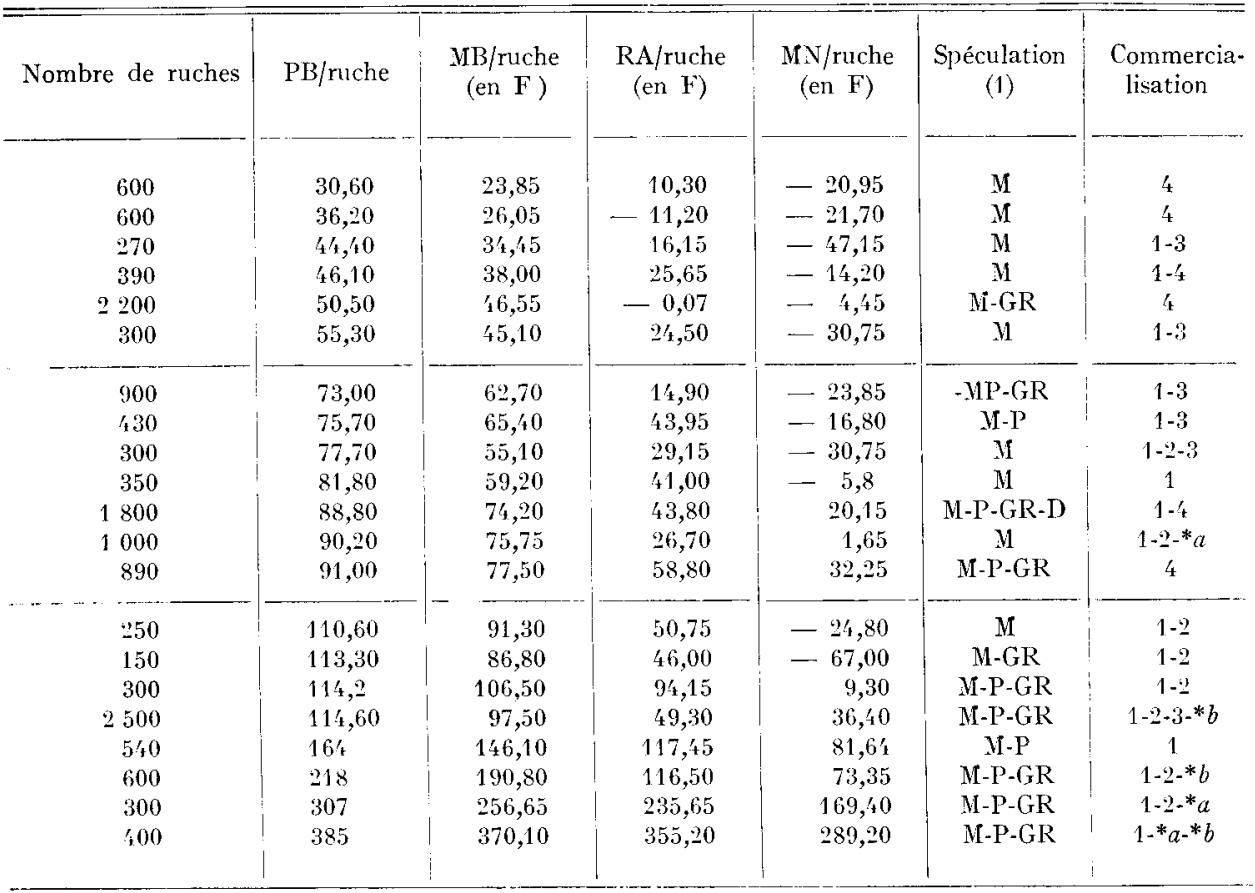

(1) M : Miel P : Pollen GR : Gelée royale D : Divers (Cire, essaims, venin, reines,...).

\section{Productivité brute du capilal d'exploitation.}

Pour l'ensemble des exploitations, la productivité brute moyenne est de $\mathbf{1 , 5 3}$. En fait, il faut bien voir qu'une grande partie du capital est amorti et qu'il faut un capital assez faible pour une exploitation apicole. Si on prend l'exemple d'une exploitation récente ( 6 ans) et très bien mécanisée, ce taux est de 0,96 , c'est-à-dire que le capital investi permet de dégager un produit équivalent en une année.

\section{Le travail.}

Il faut 4 à 5 heures de travail par ruche et par an. Sur les 2 I exploitations visitées, 6 exploitations emploient des permanents et 7 emploient des temporaires. Il n'y a que les exploitations de plus de $I$ ovo ruches qui ont des permanents. Les temporaires ne travaillent que de 2 à 5 mois. 


\section{CONCLUSION}

Cette enquête, réalisée auprès de $2 \mathrm{I}$ apiculteurs professionnels, a apporté certains éléments d'appréciation sur la rentabilité de l'activité apicole. Des aspects intéressants du fonctionnement des entreprises et des conditions de formation des résultats économiques sont aperçus. Pourtant, l'observation ne porte que sur un très petit nombre d'entreprises et ne pouvait suffire à rendre compte de tout ce qui se rapporte à une activité aussi complexe et multiforme que l'apiculture. Il y a en réalité de nombreuses apicultures professionnelles, autant qu'il y a de milieux de production, de formes d'activité, de dimensions d'entreprise, de modes de commercialisation. Il était inévitable que chacun de ces aspects ne puisse être étudié suffisamment dans le cadre de cette enquête.

De l'analyse comparative des entreprises, on retiendra essentiellement :

I. La grande variabilité des produits, des marges et des revenus des apiculteurs. On constate que le produit d'une ruche peut varier du simple au décuple suivant le choix des activités et le procédé de vente. Les écarts sont plus considérables encore pour les marges brutes et les revenus nets. Il existe des entreprises dont les revenus sont négatifs ou à peine suffisants pour assurer une rémunération normale du travail de l'entrepreneur ; mais il existe aussi des entreprises dont les profits sont très appréciables, supérieurs à too $\mathrm{F}$ par ruche, et où le taux de rentabilité des capitaux d'exploitation peut atteindre de $\mathrm{I} 5$ à $20 \mathrm{p}$. Ioo.

2. Le poids important des charges fixes et spécialement des charges de travail dans l'ensemble des charges. - Les facteurs variables jouent un rôle assez secondaire dans cette activité très exigeante en main-d'œuvre permanente.

3. L'effet d'échelle très peu marqué sur l'économie des charges. - Cela provient sans doute du caractère spécifique de l'activité apicole. On peut considérer l'entreprise apicole comme une manufacture dont les techniques de travail sont sensiblement les mêmes que le rucher ait 300 ou 2500 ruches. Il est intéressant de noter à ce sujet, qu'il semble, d'après l'étude, qu'il existe certains seuils de dimension qui seraient des multiples de 300 et qui seraient en relation avec les besoins en travail.

4. L'incidence considérable sur les résultats économiques des formes de production et des modes de commercialisation. - Il apparaît clairement que la rétussite économique est liée à une diversification de la production combinée à une recherche de la vente directe pour une fraction au moins de la production.

L'ensemble de ces résultats fait donc apparaître que tout jugement sur la profession apicole ne peut être que nuancé. On doit se garder de penser que, dans la conjoncture actuelle de faiblesse des prix de vente du miel, ou dans la perspective défavorable cle renchérissement des coûts du travail, le métier d'apiculteur est un métier fini. Il existe encore des situations présentant des rentes appréciables de production ou de commercialisation. Aussi bien cette étude a-t-elle montré l'intérêt qu'il y aurait à disposer d'une information plus complète sur plusieurs aspects importants liés au développement économique de la branche apicole : 
- l'organisation du travail et la notion de seuil de dimension qui est encore mal dégagée ;

— les productions spéciales (pollen, gelée royale, hydromel, pollinisation...);

- la commercialisation des produits apicoles.

Reçu pour publication en mai 1969.

\author{
SUMMARY
}

ECONOMIC STUDY OF SOME BEE-KEEPING UNITS IN THE " BASSIN PARISIEN"

Twenty-one professional bee-keeping units, all located north of the river Loire, have been submitted to an economic study dealing with the accountancy of the companies and on the information provided by the apiculturists who accepted this inquiry. The results obtained show the great variability of the products, of the additional cover and income of the apiculturists, the heavy burden of fixed working expenses and especially of the cost of labour in the total expenses; they also bring out that the bigger size of a bee-keeping unit ("scale effect ") hardly allows any retrenchment and that the production forms and marketing methods considerably affect the economic results obtained.

Therefore any judgement on the bee-keeping profession must be very moderate. In spite of the difficulties encountered now, because of the low selling prices of honey, in some cases rents of production and marketing are still high enough.

\title{
ANNEXE I
}

FRAIS D'ENTRE'TIEN D'UNE RUCHE

A partir de données prises dans 4 exploitations il a été possible de définir les frais d'entretien d'une ruche.

Les ruches sont passées au carbonyl tous les cinq ans et pendant cette période les cadres sont tous changés. La ruche est du type Dadant r2 cadres.

I 2 cadres de corps de ruche $\ldots \ldots \ldots \ldots \ldots \ldots \ldots, 8,52$

r2 cadres de hausse $\ldots \ldots \ldots \ldots \ldots \ldots \ldots \ldots \ldots \ldots \ldots, 6,36$

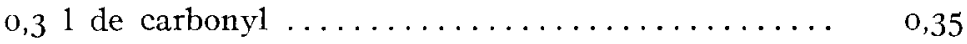

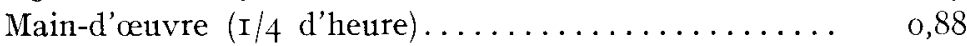

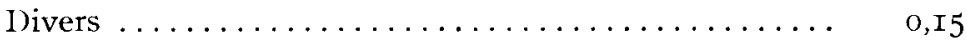

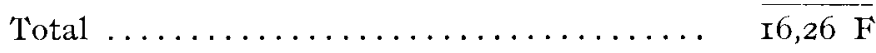

soit par ruche et par an $: 3,25 \mathrm{~F}$.

La méthode qui consiste à paraffiner les ruches est onéreuse aut départ mais l'entretien est pratiquement nul ensuite. On ne possède pas de données chiffrées concernant cette méthode. 


\section{ANNEXE 2}

COUंTS DE PRODUCTION DU MIEL,

Il paraît possible de définit un coût de production du miel à partir des données concernant 9 exploitations ne pratiquant aucune autre spéculation. Le calcul porte sur une production totale de 70 tonnes de miel provenant de 3730 ruches (moyenne : I $8,7 \mathrm{~kg}$ ). Le total des charges globales s'élève à $309200 \mathrm{~F}$, ce qui donne un coût total moyen de production de $4,40 \mathrm{~F}$ par $\mathrm{kg}$ de miel.

Les coûts de production se répartissent comme suit :

Coûts fixes réels ........... I07 300 F soit $\mathrm{I}, 53 \mathbf{F} / \mathrm{kg}$

Cồts variables réels.......... $\quad 3^{8} 6 \mathrm{I}_{5} \mathrm{~F}$ soit $0,55 \mathrm{~F} / \mathrm{kg}$

Côtuts fixes calculés .......... I 45 I Io $\mathrm{F}$ soit $2,08 \mathrm{~F} / \mathrm{kg}$

Cotits variables calculés ........ I7 075 $\mathrm{F}$ soit $0,24 \mathrm{~F} / \mathrm{kg}$

Les cô̂ts de production varient d'une exploitation à l'autre. L'apiculteur qui a les coûts les plus faibles $\left(2,9^{8} \mathrm{~F} / \mathrm{kg}\right)$ tient une comptabilité et il semble possible de retenir cette valeur calculée pour une bonne année de récolte.

Il n'est pas possible d'analyser les coûts de production dans toutes les exploitations faute de données suffisamment précises. A titre indicatif on peut signaler les valeurs suivantes qui ont été trouvées : $2,98,4,40,4,70,5$, I $5,5,50,6,50,8,90,9,70$. 